\title{
Effects of cardiac resynchronization therapy on health-related quality of life in older adults with heart failure
}

\author{
Karin $\mathrm{F} \mathrm{Hoth}{ }^{1,2}$ \\ Justin $\mathrm{Nash}^{3}$ \\ Athena Poppas ${ }^{4}$ \\ Kristin E Ellison ${ }^{4}$ \\ Robert $\mathrm{H} \mathrm{Paul}^{5}$ \\ Ronald A Cohen ${ }^{3}$ \\ 'Division of Psychosocial Medicine, \\ National Jewish Medical and \\ Research Center, Denver, CO, \\ USA; ${ }^{2}$ Department of Psychiatry, \\ University of Colorado, Denver, CO, \\ USA; ${ }^{3}$ Department of Psychiatry \\ and Human Behavior; ${ }^{4}$ Department \\ of Cardiology, Warren Alpert \\ Medical School of Brown University, \\ Providence, RI, USA; ${ }^{5}$ Department \\ of Psychology, Division of Behavioral \\ Neuroscience, University of Missouri \\ St. Louis, St. Louis, MO, USA
}

Correspondence: Karin F. Hoth I 400 Jackson St, Denver, CO 80206, USA

$\mathrm{Tel}+\mathrm{I} 303398$ II 39

Fax +13032702143

Email hothk@njc.org

\begin{abstract}
The aim of the study was to examine changes in health-related quality of life among older adults undergoing cardiac resynchronization therapy (CRT), a pacemaker based intervention for heart failure. Twenty-one patients (mean age $=68 ; \mathrm{SD}=8$ ) completed measures of cardiovascular health and quality of life prior to and 3-months post-CRT. Following the intervention, patients reported improvements in their perception of their physical functioning $(\mathrm{t}=2.8, p<0.01)$ and feelings of vitality $(\mathrm{t}=2.9, p<0.01)$ on the MOS SF-36 Health Survey. Patients improved on objective clinical measures of exercise capacity, cardiac ejection fraction, and ventricular dyssynchrony. Younger patients reported greater improvements in physical functioning and decreases in pain. Higher baseline body mass index was associated with less improvement in physical functioning. Finally, patients with nonischemic heart failure reported greater improvements on multiple subscales of the SF-36 than patients with ischemic heart failure. This preliminary study documented improvements in health-related quality of life following CRT. The findings highlight that specific patient characteristics may be associated with quality of life changes. Future studies will benefit from including quality of life measures that assess multiple health-related domains.
\end{abstract}

Keywords: cardiac resynchronization therapy, heart failure, quality of life

\section{Introduction}

Heart failure is one of the leading chronic illnesses that impacts quality of life (Alonso et al 2004). In addition to decreasing general well-being, there is evidence that poor health-related quality of life is a predictor of subsequent hospital admission and death among elderly patients with heart failure (Ho et al 2005; Rodriguez-Artalejo et al 2005). The high prevalence and broad impact of heart failure underscores the importance of understanding how treatment interventions relate to patient outcomes, including improvements in quality of life.

Cardiac resynchronization therapy (CRT) is a recently developed pacing therapy for patients with refractory heart failure who demonstrate symptomatic ventricular dyssynchrony. CRT has been shown to improve heart failure-specific quality of life, along with multiple clinical endpoints including heart failure symptoms, exercise capacity, hospitalization rates, and longevity (Abraham 2003; Bradley et al 2003). Heart failure-specific quality of life, as measured by the Minnesota Living with Heart Failure Questionnaire (LHFQ), reflects self-reports of the extent to which heart failure symptoms prevent individuals from living as they would like (Rector et al 1987a). Heart failure-specific quality of life combines physical, socioeconomic, and psychological impairments into a single dimension. Improvements in heart failure-specific quality of life have been observed from one month to one year following CRT (Cazeau et al 2001; Abraham et al 2002; Gras et al 2002; Abraham 2003). 
Relatively little is known about the impact of CRT on different domains of more general health-related quality of life. Health-related quality of life, as assessed by the MOS SF-36, is not disease specific and captures quality of life in multiple domains (ie, physical functioning, pain, social functioning, mental health, and general health perceptions). Patients with heart failure experience decline in multiple aspects of quality of life, not just physical functioning (Hobbs et al 2002). Among the few trials examining the impact of CRT on health-related quality of life, Skobel and colleagues (2005) reported that CRT produced improvements in multiple domains. The improvements were particularly evident in those with Cheyne-Stokes respiration (CSR). This finding illustrates that patients with different clinical characteristics may experience differential changes in quality of life following CRT.

Factors that might relate to improvements in quality of life following CRT remain unidentified. In one study that focused on heart failure-specific quality of life, Krahn and colleagues (2002) examined several patient characteristics to explain why some patients undergoing CRT demonstrated improvement in their heart failure symptoms while others did not. The authors examined age, sex, heart failure etiology, QRS width, ejection fraction, and heart failure severity (ie, New York Heart Association Class I-IV; higher classes reflect greater physical limitation). No associations were found between quality of life improvement and these factors. Despite this negative finding in heart failure-specific quality of life, it is possible that there are factors that relate to improvements in other domains of health-related quality of life. For example, certain patients may be more likely to perceive improvements in their general physical health, social functioning, or emotional functioning following CRT than other patients.

The aim of the current study was to examine changes in health-related quality of life following CRT among older adults with Class II-IV heart failure. We sought to extend previous findings of improvement in heart failure-specific quality of life by examining quality of life in a broader range of health-related domains. A secondary objective of the study was to identify patient characteristics that might relate to improvement in specific quality of life domains.

\section{Methods}

\section{Study sample}

The current study included 24 older adults with heart failure who were candidates for cardiac resynchronization therapy. Patients were recruited through the arrhythmia services division of cardiology in a major teaching hospital. All participants were enrolled in a larger, prospective study of physiological and psychological changes following cardiac resynchronization therapy. To be enrolled in the study, individuals were required to be over the age of 55, have NYHA Class II-IV heart failure, cardiac ejection fraction below $35 \%$, and QRS duration greater than or equal to $130 \mathrm{~ms}$. Consistent with aims of the parent study, exclusion criteria were as follows: 1) previous diagnosis with a cognitive disorder including dementia or mild cognitive impairment, 2) neurological disorder, 3) major psychiatric disorder such as schizophrenia, bipolar illness, or substance abuse, or 4) current untreated major depressive episode.

Of the 24 participants who completed the baseline assessment, 3 did not return for follow-up. During the follow-up window, 2 participants experienced lead displacement and underwent revision of their device. One of these individuals was included in the follow-up and one reported being too tired to return for the final study visit. The other participant who withdrew from the study indicated that he was unable to return due to transportation concerns. Overall, this represents an $88 \%$ retention rate. Data analyses were based upon the final sample of 21 participants with complete baseline and 3-month follow-up data. The demographic and clinical characteristics of the sample are presented in Table 1.

\section{Procedure}

The study was approved by the local Institutional Review Board and all patients provided written informed consent before enrollment. The baseline study visit was conducted within the 2 weeks before implantation of the biventricular (BiV) pacemaker/implantable cardioverter defibrillator (ICD; note: 1 patient received a BiV device without a defibrillator). During the baseline visit, participants completed a medical history interview. Medical history variables were coded as either present or absent according to participants' self-report and were confirmed by a medical chart review. Medical and cardiovascular health was assessed using electrocardiogram, echocardiogram, and the 6-minute walk test (ATS Board of Directors 2002). The baseline echocardiogram, 6-minute walk test, and quality of life measures were completed during the baseline study visit within 2 weeks of the implant. All other clinical measures were obtained within the month before the implant and were collected via medical chart review. The follow-up echocardiogram, 6-minute walk test, and quality of life measures were completed during a study visit approximately 3-months after BiV/ICD implantation. The average length of follow-up after BiV/ICD implantation was 89 days $(\mathrm{SD}=13)$. 
Table I Baseline demographic and clinical characteristics of sample

\begin{tabular}{|c|c|c|c|}
\hline Patient characteristics & Percent & Mean (SD) & Range \\
\hline \multicolumn{4}{|l|}{ Demographics } \\
\hline Age (years) & & $67.9(8.4)$ & $56-84$ \\
\hline Sex (\% female) & $19 \%(4 / 21)$ & & \\
\hline Education (years) & & $12.2(3.7)$ & $5-18$ \\
\hline \multicolumn{4}{|l|}{ Baseline lab measures } \\
\hline Systolic BP (mmHg) & & I28.7 (|3.9) & $108-160$ \\
\hline Diastolic BP (mmHg) & & $73.9(9.8)$ & $60-90$ \\
\hline Body mass index $\left(\mathrm{kg} / \mathrm{m}^{2}\right)$ & & $24.2(6.9)$ & $|3.8-4| .6$ \\
\hline Blood glucose (mg/dl) & & $\mid 40.6(5 \mid .2)$ & $91-262$ \\
\hline Blood urea nitrogen $(\mathrm{mg} / \mathrm{dl})$ & & $18.2(9.2)$ & $7-4 \mid$ \\
\hline Creatinine $(\mathrm{mg} / \mathrm{dl})$ & & I.I (0.3) & $0.6-1.7$ \\
\hline Beta natriuretic peptide $(\mathrm{pg} / \mathrm{ml})$ & & $205.3(238.9)$ & 16.7-939.7 \\
\hline \multicolumn{4}{|l|}{ Medical history information } \\
\hline Hypertension & $67 \%(|4 / 2|)$ & & \\
\hline Hypercholesterolemia & $71 \%(15 / 21)$ & & \\
\hline Type 2 diabetes mellitus & $38 \%(8 / 2 I)$ & & \\
\hline History of smoking & $67 \%(|4 / 2|)$ & & \\
\hline Prior myocardial infarction & $52 \%(11 / 2 I)$ & & \\
\hline History of atrial fibrillation & $33 \%(7 / 21)$ & & \\
\hline Percutaneous coronary intervention & $52 \%(\mid I / 2 I)$ & & \\
\hline Coronary artery bypass surgery & $14 \%(3 / 21)$ & & \\
\hline Previous ICD & $10 \%(2 / 21)$ & & \\
\hline Valve surgery & $5 \%(I / 2 I)$ & & \\
\hline \multicolumn{4}{|l|}{ Heart failure etiology } \\
\hline Ischemic & $48 \%(10 / 21)$ & & \\
\hline Nonischemic & $52 \%(11 / 2 I)$ & & \\
\hline \multicolumn{4}{|l|}{ NYHA Heart Failure Class } \\
\hline Class II & $55 \%(|I / 2|)$ & & \\
\hline Class III and IV* & $45 \%(9 / 21)$ & & \\
\hline \multicolumn{4}{|l|}{ Medications } \\
\hline Beta blockers & $95 \%(20 / 21)$ & & \\
\hline Aspirin/antithrombotics & $81 \%(|7 / 2|)$ & & \\
\hline Lipid lowering agents & $76 \%(|6 / 2|)$ & & \\
\hline ACE inhibitors & $76 \%(16 / 21)$ & & \\
\hline Diuretics & $67 \%(14 / 21)$ & & \\
\hline Digoxin & $43 \%(9 / 21)$ & & \\
\hline Hypoglycemics & $33 \%(7 / 21)$ & & \\
\hline Antidepressants/antianxiety & $33 \%(7 / 21)$ & & \\
\hline Vasodilators & $29 \%(6 / 2 I)$ & & \\
\hline
\end{tabular}

Notes: *Class III and IV were grouped for analytic purposes because I person was Class IV.

\section{Measures}

\section{Electrocardiogram}

Electrocardiogram was used to quantify the degree of intraventricular dyssynchrony. QRS complex duration expressed in milliseconds was the primary variable of interest.

\section{Echocardiogram}

Two-D transthoracic echocardiograms were performed with an IE33 machine (Philips, Andover, MA) according to American Society of Echocardiography standards. Left ventricular ejection fraction was calculated based upon biplane volumes. (EF $=\mathrm{EDV}-\mathrm{ESV} / \mathrm{EDV})$. The biplane method of discs utilized two orthogonal views from the apex; it calculated volumes from summation of areas from diameters of 20 cylinder discs of equal height. The discs were apportioned by dividing the chamber's longest length into 20 equal sections. In 3 participants, a single plane method from the apical 4-chamber view was employed due to suboptimal image quality.

\section{6-minute walk test}

The 6 minute walk test was used as a measure of functional capacity. It has been widely used to measure the response to interventions in moderate to severe pulmonary and cardiac 
disease (Enright 2003). The test was administered consistent with the American Thoracic Society guidelines (ATS Board of Directors 2002). Total distance walked in feet in six minutes was the outcome measure.

\section{Beck depression inventory-II (BDI-II)}

The BDI-II (Beck et al 1996) is a 21-item self-report questionnaire measuring severity of depressive symptoms. Respondents were asked to endorse statements characterizing their mood. The maximum total score is 63 , with scores greater than 14 suggesting clinically significant depressive symptoms.

\section{Minnesota living with heart failure questionnaire (LHFQ)}

The LHFQ is a 21-item self-report measure (Rector et al 1987b). Respondents were asked to rate the extent to which heart failure specific symptoms prevented them from living as they wanted within the past month on a 6-point Likert scale (Rector and Cohn 1992; Bennett et al 2002).

\section{Medical outcome study short-form general health survey (SF-36)}

The SF-36 is a 36-item health status and quality of life inventory (Ware and Sherbourne 1992) developed to measure health concepts across different age, disease, and treatment groups. Respondents indicated the degree or frequency of problematic health, behavioral, and emotional events on a Likert scale. The SF-36 assesses health status in eight areas: physical functioning, role disruption due to physical difficulties, role disruption due to emotional difficulties, social functioning, mental health, vigor, health perception, and pain. Scores on the subscales range from 0 to 100 . The measure has good validity and reliability and has been extensively used in healthy and medical populations (McHorney et al 1993).

\section{Data analysis}

Descriptive statistics regarding the sample demographic and medical characteristics were calculated and are presented in Table 1. The primary analysis to examine change in health-related quality of life included eight paired t-tests to compare patients' self-reported quality of life on each of the SF-36 scales at baseline and 3-months post-CRT. To examine change in the patients' heart failure symptoms and cardiovascular health, paired t-tests were calculated comparing baseline and follow-up values on the LHFQ, 6 minute walk test, ejection fraction, and QRS duration form electrocardiogram.
A secondary aim of the study was to examine individual difference variables that might be associated with changes in quality of life following CRT. Age, body mass index (BMI), heart failure severity measured by NYHA heart failure class (ie, Class II vs. Class II and IV), heart failure etiology (ie, ischemic vs nonischemic), change in ejection fraction and QRS duration from baseline to follow-up were examined. For the secondary analyses, the eight SF-36 subscale scores were converted into standardized z-scores. Baseline z-scores were subtracted from follow-up z-scores to create a change score for each SF-36 subscale. Thus, positive change scores indicate improvement in quality of life after CRT and negative change scores indicate a decline in quality of life following CRT. Bivariate correlations were calculated between the continuous variables (ie, age, BMI, change in ejection fraction and QRS duration) and SF-36 change scores. Independent samples t-tests were used to compare change in quality of life for the dichotomous variables (ie, heart failure etiology and NYHA class). Data were analyzed using SPSS 11.0 software. Given the exploratory nature of the study, an alpha level of 0.05 was set a priori for all analyses.

\section{Results}

Paired t-tests comparing baseline and follow-up scores on the SF-36 for the entire sample revealed that patients reported statistically significant improvements on the physical function $(\mathrm{t}=2.8, \mathrm{df}=20, p<0.01)$ and vitality $(\mathrm{t}=2.9, \mathrm{df}=20, p<0.01)$ subscales (see Table 2$)$. The decline in LHFQ score approached statistical significance $(\mathrm{t}=1.9, p<0.07)$. Consistent with the inclusion criteria, the average BDI-II score for the sample suggested minimal symptoms of depression at baseline and follow-up. Regarding the clinical measures, paired t-tests showed that there was a significant increase in 6-minute walk distance (1249 $\mathrm{ft}$ vs $1150 \mathrm{ft} ; \mathrm{t}=1.9, \mathrm{df}=18, p=0.02)$, increase in ejection fraction $(30 \%$ vs 39\%; $\mathrm{t}=3.9$, df $=20, \mathrm{p}<0.01)$, decrease in end diastolic (174 vs 146; $\mathrm{t}=3.8, \mathrm{df}=15, p<0.01)$ and systolic volumes (123 vs $94 ; \mathrm{t}=6.2, \mathrm{df}=15, p<0.001)$, and decrease in QRS duration (162 vs 143; $\mathrm{t}=3.0, \mathrm{df}=20$, $p=0.001$ ) following CRT.

The secondary analyses to examine the potential impact of clinical characteristics on change in health-related quality of life revealed differential changes in quality of life for several variables. Correlation analyses revealed that age was significantly associated with change on the physical function subscale $(\mathrm{r}=-0.65, p<-0.01)$ and the pain subscale $(\mathrm{r}=-0.58, p<0.01)$. Specifically, younger patients tended to report greater improvement in physical functioning and 
Table 2 Change on clinical and psychosocial measures following CRT

\begin{tabular}{|c|c|c|c|c|c|c|}
\hline \multirow[t]{2}{*}{ Measure } & \multicolumn{3}{|c|}{$\begin{array}{l}\text { Total sample mean } \\
\text { score }(S D)(n=21)\end{array}$} & \multirow[t]{2}{*}{$\begin{array}{l}\text { Nonischemic HF } \\
\text { z-score change }(n=I I)\end{array}$} & \multirow[t]{2}{*}{$\begin{array}{l}\text { Ischemic H } \\
\text { z-score change }(n=10)\end{array}$} & \multirow[t]{2}{*}{ Sig. } \\
\hline & Baseline & Follow-up & Sig. & & & \\
\hline \multicolumn{7}{|l|}{ SF-36 } \\
\hline Physical function & $56.2(23.7)^{* *}$ & $65.5(24.8)^{* *}$ & $t=2.8$ & $+0.7^{*}$ & $+0.1^{*}$ & $t=2.3$ \\
\hline Role-physical function & $52.5(4 \mid .3)$ & $58.7(40.8)$ & & +0.6 & -0.3 & \\
\hline Pain index & $69.0(24.1)$ & $75.9(22.5)$ & & +0.6 & -0.1 & \\
\hline General health perception & $55.5(22.7)$ & $64.1(21.7)$ & & $+0.9 *$ & $-0.2^{*}$ & $t=2.3$ \\
\hline Vitality score & $5 I . I(I 8.6)^{* *}$ & $64.0(23.6)^{* *}$ & $t=2.9$ & $+1.19 * *$ & $-0.1 * *$ & $t=2.8$ \\
\hline Social function & $81.5(22.9)$ & $82.7(26.4)$ & & $+0.6 *$ & $-0.6 *$ & $t=2.1$ \\
\hline Role-emotional function & $63.3(38.8)$ & $70.0(35.7)$ & & +0.6 & -0.2 & \\
\hline Mental health index & $74.5(16.3)$ & $80.6(17.8)$ & & +0.4 & +0.3 & \\
\hline MLHF questionnaire & $30.6(24.3)$ & $21.7(23.9)$ & & & & \\
\hline Beck Depression Inventory-II & $7.3(6.4)$ & $5.8(6.5)$ & & & & \\
\hline \multicolumn{7}{|l|}{ Echocardiogram } \\
\hline Ejection fraction (\%) & $30.0(8.8)^{* * *}$ & $39.2(9.0)^{* *}$ & $t=5.4$ & & & \\
\hline LV end diastolic volume & $174.1(76.2)^{* *}$ & $146.3(74.5)^{* *}$ & $t=3.8$ & & & \\
\hline LV end systolic volume & $123.6(72.4)^{* * *}$ & $94.1(67.2)^{* * *}$ & $t=6.2$ & & & \\
\hline \multicolumn{7}{|l|}{ Electrocardiogram } \\
\hline QRS duration (ms) & $163.4(17.0)^{* * *}$ & I44.I (I 4.5$)^{* * *}$ & $t=3.9$ & & & \\
\hline 6-Minute Walk Test (feet) & $1150.0(279.1)^{*}$ & $1248.9(345.2)^{*}$ & $t=2.7$ & & & \\
\hline
\end{tabular}

Notes: ${ }^{*} \mathrm{p}<0.05,{ }^{*} \mathrm{p}<0.01$.

Abbreviations: LV, left ventricular; HF, heart failure.

greater decreases in bodily pain following CRT (see Table 3 ). Figure 1 illustrates the association between age and changes in physical function. Correlation analysis also revealed that higher baseline BMI was associated with significantly less improvement in physical functioning following CRT $(\mathrm{r}=-0.44, p<0.05)$. Change in ejection fraction and change in QRS duration were not significantly associated with change on any of the quality of life scales. Patients with nonischemic heart failure reported greater improvements on the physical function, vitality, general health perception, and social function subscales following CRT than patients with ischemic heart failure (see Table 2). Finally, patients with Class II heart failure at baseline reported greater improvement on the mental health subscale following CRT than patients with Class III and IV heart failure at baseline ( +0.8 vs. -0.2 $\mathrm{z}$-score change; $\mathrm{t}=2.5, \mathrm{df}=19, p<0.05)$.

\section{Discussion}

The results of the current study provide two important advances to the current literature on quality of life following CRT. First, the present findings suggest that on average, CRT improves general health-related quality of life, particularly in

Table 3 Intercorrelations between clinical variables and change in health-related quality of life domains

\begin{tabular}{lllcl}
\hline MOS SF-36 domain change & Age & Baseline body mass index & Ejection fraction change & QRS duration change \\
\hline Physical function & $-0.65^{* *}$ & $-0.44^{*}$ & 0.11 & -0.21 \\
Role-physical function & -0.10 & 0.11 & -0.03 & -0.15 \\
Pain index & $-0.58^{* *}$ & -0.20 & 0.01 & -0.37 \\
General health perception & -0.19 & 0.04 & -0.12 & -0.25 \\
Vitality & -0.26 & 0.03 & -0.06 & -0.30 \\
Social function & -0.19 & 0.03 & -0.22 & -0.31 \\
Role-emotional function & -0.21 & 0.24 & 0.01 & -0.21 \\
Mental health index & -0.09 & 0.01 & -0.15 & -0.16
\end{tabular}

Notes: $* p<0.05, * * p<0.01$. 


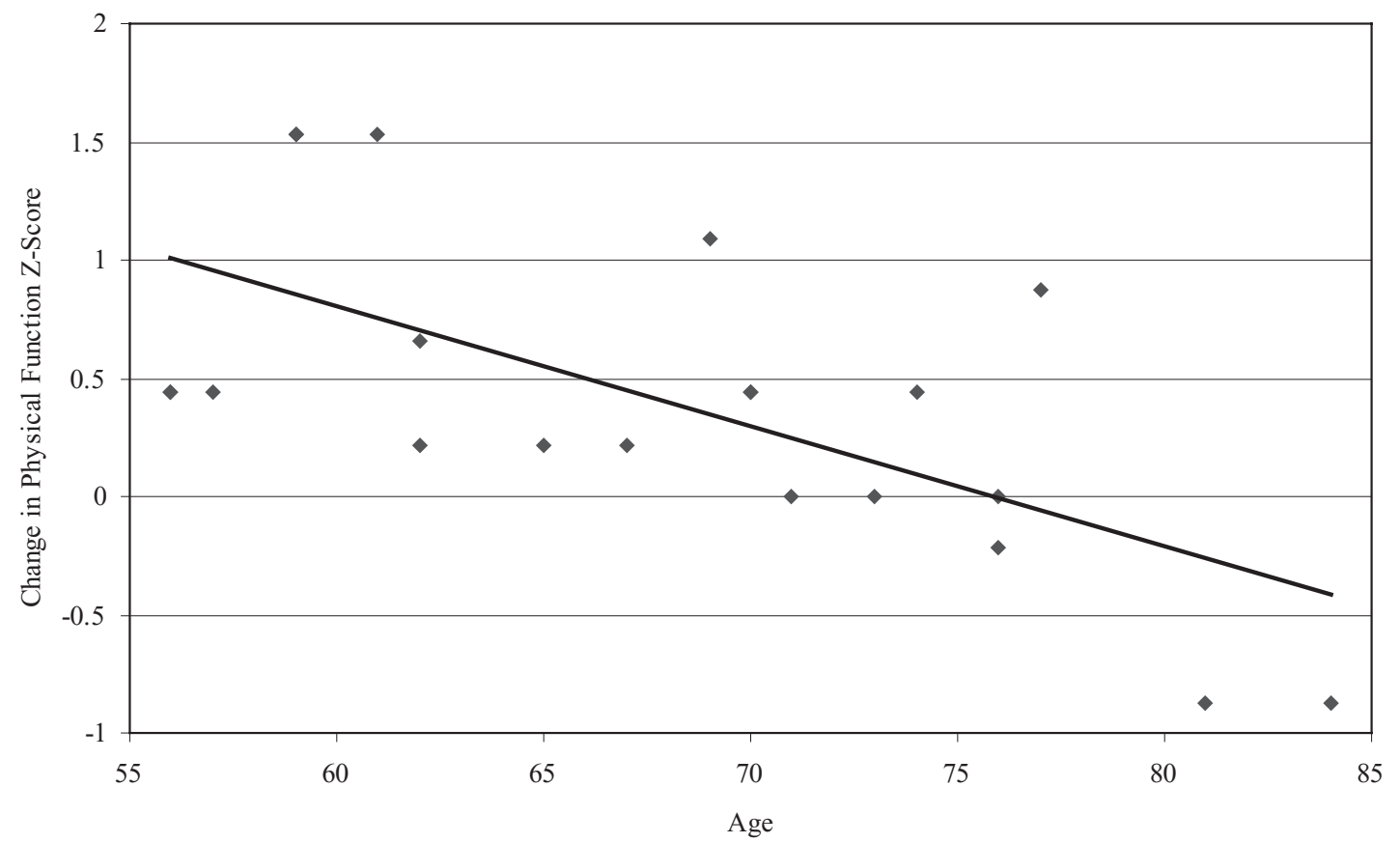

Figure I Intercorrelation between age and change in SF-36 Physical Function Score.

the domains of physical functioning and feelings of vitality. Additionally, the results suggest that the areas of quality of life that improve may depend on, or at least relate to, certain patient characteristics. That is, certain subsets of patients may be more likely to experience quality of life improvements than others.

Younger patients were more likely to perceive an improvement in physical functioning and level of pain than older patients after CRT. This finding is consistent with the results of a recent study of pacemaker implantation in patients with arrhythmia (Fleischmann et al 2006). Fleischmann and colleagues (2006) found that patients older than 75 years experienced less improvement in physical functioning than younger subjects. Several clinical trials of CRT have examined age effects on clinical measures of heart failure and have observed similar benefits in mortality, ventricular dyssynchrony and ejection fraction for patients above and below the median age of the sample (eg, Cleland et al 2005, cut at age 66; Bleeker et al 2005, cut at age 70); thus, it is notable that we observed age effects in relation to general health-related quality of life in the current study of CRT. We examined age as a continuous variable rather than using an age cutoff, and this may have increased our ability to detect age effects on perceived improvement in health-related quality of life.

Patients with nonischemic heart failure reported greater improvements on a number of domains of health-related quality of life (ie, physical function, vitality, general health perception, and social function) than patients with ischemic heart failure. Patients with higher BMIs at baseline reported less improvement in their physical functioning. Although it is not possible to determine the reason for these differences based upon the current data, one potential hypothesis is that patients with ischemic heart failure and higher BMIs have additional medical concerns that serve to maintain poorer general health-related quality of life after CRT.

We also examined the potential association between change in cardiac ejection fraction and QRS duration after CRT and improvements in quality of life; however, these variables were not significantly associated with change on any of the quality of life subscales. Post-hoc examination of the data revealed that ejection fraction and QRS duration also was not associated with self-reported quality of life at the baseline assessment. It is possible that the small sample size limited our ability to identify a potential association, but this is unlikely, since physiological measures such as ejection fraction and mitral valve regurgitation have not been associated with patients' perceived quality of life in several previous studies (Rector and Cohn 1992; Hauptman et al 2006). Larger longitudinal studies are needed to confirm the lack of an association, but the finding may reflect the fact that patient perceptions of function are complex and likely determined by interactions between individual differences in behavioral, cognitive, and emotional factors and disease severity. 
By design, the study included patients with Class II-IV heart failure who were free of neurological and major psychiatric disorders. Several previous trials of CRT have focused on patients with Class III and IV heart failure; thus, our sample is somewhat healthier than these previous trials. Replication of the current findings in a sample including more patients with Class IV heart failure will be helpful to strengthen the generalizability of the findings. Importantly, the inclusion of patients with Class II heart failure allowed us to examine changes in quality of life comparing patients with Class II and Class III/IV heart failure at baseline. Patients with Class II heart failure reported greater improvement on social functioning than those rated as Class III and IV, and there were no significant differences in magnitude of improvement on the other SF-36 quality of life domains. This suggests that patients with Class II heart failure benefited at least equivalently from CRT in terms of their perceived improvement in general health-related quality of life.

The current study had some limitations that are important to consider. First, the sample size was fairly small, and as a result the findings should be considered preliminary. The study was designed with a 3 month follow-up based on previous research suggesting that improvements in heart failure-specific quality of life have been observed as early as 1-3 months (Cazeau et al 2001; Abraham et al 2002; Gras et al 2002). The current findings could have been extended by following patients for a longer period of time to examine the long-term effects of CRT on health-related quality of life. However, the 3 month time period coincides with significant improvement in cardiac function following CRT (Abraham 2003), suggesting that this time period is appropriate to observe effects on health-related quality of life.

Although previous large clinical trials of CRT have included brief measures of quality of life, typically defined using the LHFQ, the current study is among the first to examine changes in the more general construct of healthrelated quality of life. We found that patient perceptions of their physical functioning and vitality improved 3 months after CRT. Given the specific focus on changes in quality of life after CRT, we analyzed several clinical variables in relation to changes in quality of life and found that age, baseline BMI, and heart failure etiology (ie, ischemic vs. nonischemic) were associated with differential improvement in health-related quality of life. The fact that several patient characteristics were associated with changes in quality of life following CRT points to the importance of examining patient characteristics such as age and medical comorbidities that may predict who will experience the greatest improvement in their perceived health, vitality and social functioning. Larger clinical trails should include broader measures of quality of life, in order to elucidate the impact of CRT on aspects of patient well-being that go beyond the patients' perception of how heart failure specific symptoms impact their functioning.

\section{Acknowledgments}

This study was supported in part by grant AG026850 awarded to Karin Hoth, Ph.D. The authors thank Andrew Sokobin R.D.C.S. and Youngsoo Cho, M.D. for their assistance with data collection. The authors report no conflicts of interest.

\section{References}

Abraham WT. 2003. Cardiac resynchronization therapy: a review of clinical trials and criteria for identifying the appropriate patient. Rev Cardiovasc Med, 4:30-7.

Abraham WT, Fisher WG, Smith AL, et al. 2002. Cardiac resynchronization in chronic heart failure. N Engl J Med, 346:1845-53.

Alonso J, Ferrer M, Gandek B, et al. 2004. Health-related quality of life associated with chronic conditions in eight countries: results from the International Quality of Life Assessment (IQOLA) Project. Qual Life Res, 13:283-98.

ATS Board of Directors. 2002. ATS Statement: Guidelines for the six-minute walk test. Am J Respir Crit Care Med, 166:111-17.

Beck AT, Steer RA, Brown GK. 1996. BDI-II Manual. Fort Worth, TX: The Psychological Corporation.

Bennett SJ, Olridge NB, Eckert GJ, et al. 2002. Discriminant properties of commonly used quality of life measures in heart failure. Qual Life Res, 11:349-59.

Bleeker GB, Schalij MJ, Molhoek SG, et al. 2005. Comparison of effectiveness of cardiac resynchronization therapy in patients $<70$ versus $>$ or $=70$ years of age. Am J Cardiol, 96:420-22.

Bradley DJ, Bradley EA, Baughman KL, et al. 2003. Cardiac resynchronization and death from progressive heart failure: A meta-analysis of randomized controlled trails. JAMA, 289:730-40.

Cazeau S, Leclercq C, Lavergne T, et al. 2001. Effects of multisite biventricular pacing in patients with heart failure and intraventricular conduction delay. NEngl J Med, 344:873-80.

Cleland JG, Daubert JC, Erdmann E, et al. 2005. The effect of cardiac resynchronization on morbidity and mortality in heart failure. $N$ Engl J Med, 352:1539-49.

Enright PL. 2003. The six-minute walk test. Respir Care, 48:783-5.

Fleischmann KE, Orav EJ, Lamas GA, et al. 2006. Pacemaker implantation and quality of life in the Mode Selection Trial (MOST). Heart Rhythm, 3:653-9.

Gras D, Leclercq C, Tang AS, et al. 2002. Cardiac resynchronization therapy in advanced heart failure the multicenter InSync clinical study. Eur $J$ Heart Fail, 4:311-20.

Hauptman PJ, Rector TS, Wentworth D, et al. 2006. Quality of life in advanced heart failure: role of mitral regurgitation. Am Heart J, 151:213-8.

Ho PM, Masoudi FA, Peterson PN, et al. 2005. Health-related quality of life predicts mortality in older but not younger patients following cardiac surgery. Am J Geriatr Cardiol, 14:176-82.

Hobbs FD, Kenkre JE, Roalfe AK, et al. 2002. Impact of heart failure and left ventricular systolic dysfunction on quality of life: a cross-sectional study comparing common chronic cardiac and medical disorders and a representative adult population. Eur Heart J, 23:1867-76.

Krahn AD, Snell L, Yee R, et al. 2002. Biventricular pacing improves quality of life and exercise tolerance in patients with heart failure and intraventricular conduction delay. Can J Cardiol, 18:380-87. 
McHorney CA, Ware JE, Raczek AE. 1993. The MOS 36-item Short Form Health Survey (SF-36). II. Psychometric and clinical tests of validity in measuring physical and mental health constructs. Med Care, 31:247-63.

Rector TS, Francis GS, Cohn JN. 1987a. Patients' self-assessment of their congestive heart failure. Part 1 Patient perceived dysfunction and its poor correlation with maximal exercise tests. Heart Fail, 3:192-6.

Rector TS, Kubo SH, Cohn JN. 1987b. Patients' self-assessment of their congestive heart failure. Part 2: Content, reliability and validity of a new measure, the Minnesota Living with Heart Failure Questionnaire. Heart Fail, 3:198-209.

Rector TS, Cohn JN; Pimobendan Multicenter Research Group. 1992. Assessment of patient outcome with the Minnesota Living with Heart Failure questionnaire: Reliability and validity during a randomized, double-blind, placebo controlled trial of pimobendan. Am Heart J, 124:1017-25.
Rodriguez-Artalejo F, Guallar-Castillon P, Pascual CR, et al. 2005. Healthrelated quality of life as a predictor of hospital readmission and death among patients with heart failure. Arch Intern Med, 165:1274-9.

Skobel EC, Sinha AM, Norra C, et al. 2005. Effect of cardiac resynchronization therapy on sleep quality, quality of life, and symptomatic depression in patients with chronic heart failure and cheyne-stokes respiration. Sleep Breath, 9:159-66.

Ware JE, Sherbourne CD. 1992. The MOS 36-item short form health survey (SF-36). I. Conceptual framework and item selection. Med Care, 30:473-83. 